\title{
Calibration of the MCAO Canopus Bench
}

\author{
Aurea Garcia-Rissmann ${ }^{1, a}$, François Rigaut ${ }^{1}$, Matthieu Bec ${ }^{1}$, Maxime Boccas ${ }^{1}$, Ramon Galvez ${ }^{1}$, \\ Gaston Gausachs $^{1}$, Damien Gratadour ${ }^{2}$, and Benoit Neichel ${ }^{1}$ \\ 1 Gemini Observatory Southern Operations Center, c/o AURA, Casilla 603, La Serena, Chile \\ 2 LESIA - Observatoire de Paris, 5 place Jules Janssen, 92195, MEUDON, Cedex, France
}

\begin{abstract}
The final phase of implementation of all optical components, as well as their integration and tests on the Canopus MCAO bench is currently underway. We present here a detailed description of the LGS and NGS WFS calibration sequences implemented through MYST (MCAO Yorick Smart Tool), a yorick+python+glade software package developed in-house which allows multiple users to control and monitor the bench remotely over the network using EPICS ${ }^{b}$ commands. A fine tuning of the optical setup and a better understanding of the flexure/temperature dependencies is being carried out and will allow us to build the many look-up tables to be eventually used by the system (e.g. telescope primary and secondary mirrors). Preliminary work on non-common path aberrations to account for the static aberrations in the central 60 arcsec science field of view (FoV) has been done iteratively using a science focal plane wavefront sensor and has shown good results both in individual directions as well as simultaneously over the entire FoV, the latter using the tomographic approach (presented in another paper in this conference).
\end{abstract}

\section{Introduction}

The Canopus bench is the heart of the Gemini MCAO System (GeMS), and current schedule previews its first light on Gemini South telescope by mid-2010. The whole system will deliver a uniform, diffraction-limited corrected near-infrared (NIR) image quality over an extended FoV (more than 1 arcmin across). This corrected beam will then feed either Flamingos 2 or GSAOI, the next generation of instruments to be commissioned at Cerro Pachon. It is a unique and challenging project from the technological point of view and because of its control complexity. It has been designed to use the signal of 5 laser guide stars (LGS) distributed in a square asterism of 1 arcmin side (four stars at the edges and one at the center), with unprecedent demand in terms of power (50 Watt) and beam quality. Besides that, up to 3 either visible or NIR natural guide stars (NGS) will provide measurements for the correction of the tip-tilt and anisoplanatic modes, and yet another extra star in the FoV will be used for flexure corrections. When finally implemented, the F/16 beam of the Gemini telescope will be deviated in the Cassegrain instrument structure support (ISS) by the AO-fold mirror, re-directing it to the Canopus bench. The MCAO correction will then be performed by three deformable mirrors (conjugated to $0,4.5$ and $9 \mathrm{~km}$ ) and one tip-tilt mirror (TTM). A first dichroic beam splitter (BS1 in figure 1) is responsible for separating the visible from NIR light, sending the former to the wavefront sensors and the latter to the Canopus science output with a F/33.2 focal-ratio to feed the instruments. A second beam splitter (BS2) separates the visible light inside a narrow range around 589nm to illuminate the 5 LGS Shack-Hartmann wavefront sensors (SHWFS). The remaining light is sent to the tip-tilt and slow-focus sensor (SFS), which constitute the NGS WFS assembly. It consists of 3 probes, each containing a reflective pyramid that acts like a quad-cell feeding a set of 4 fibers+APDs. One of the probes contains also a small beam splitter that sends light to the SFS. The main components of the Canopus bench are shown in the figure 1, and the RTC plus servo controls are at this point almost completely integrated to work with EPICS commands. The system is currently going through a re-design of its cooling system by the mechanical engineering team, whereas the AO scientists are dedicated to optimizing the general bench control and the alignment/throughput of the optical components (see [1]

\footnotetext{
b EPICS is an Open Source software toolkit widely used at Gemini, it provides a client/server network infrastructure with publish/subscribe capabilities adapted to the distributed nature of GeMS control systems.

a e-mail: augarcia@gemini .edu
}

This is an Open Access article distributed under the terms of the Creative Commons Attribution-Noncommercial License, which permits unrestricted use, distribution, and reproduction in any noncommercial medium, provided the original work is properly cited. 


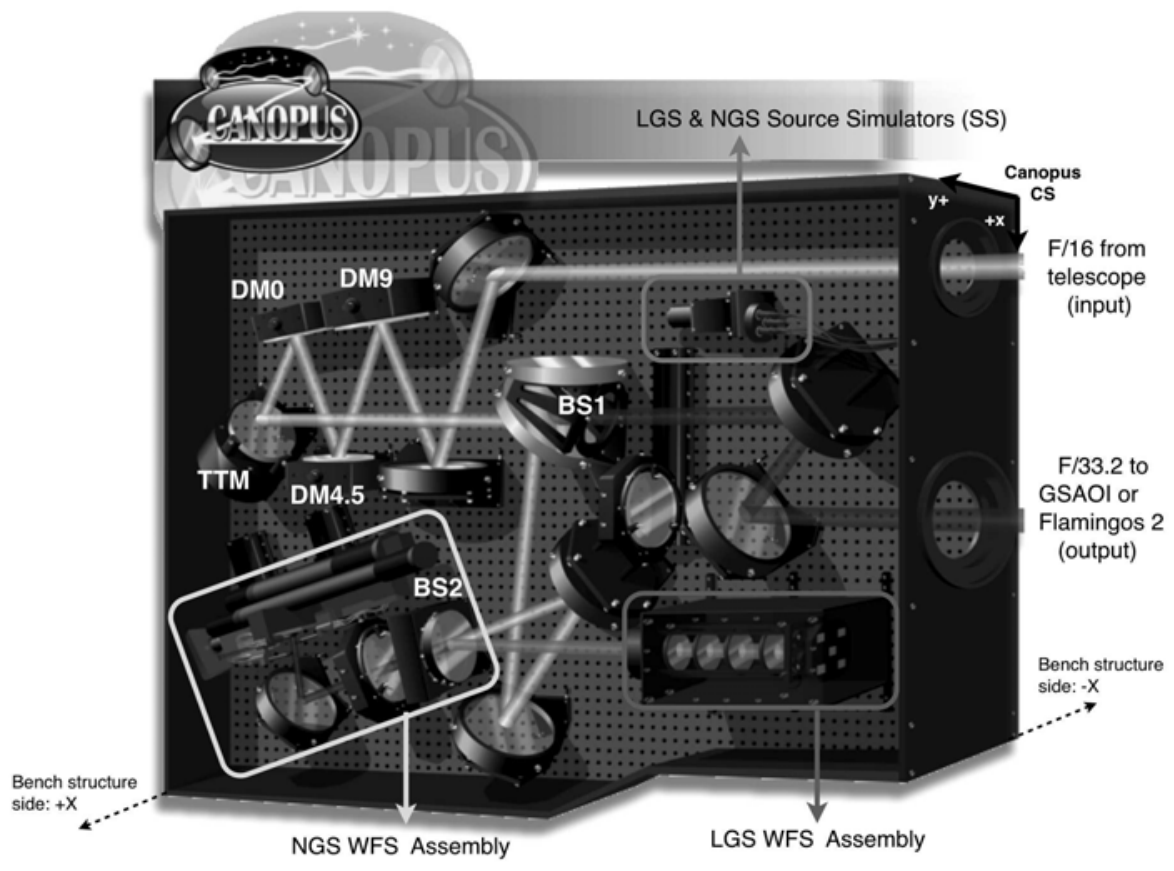

Fig. 1. Drawing showing the main components of the Canopus MCAO bench.

and [2] for more details). In this work we briefly show some aspects that are important for the optimization of the bench, such as results from acceptance tests and calibration sequences. Such routines are being implemented through the high-level software package developed by the group to make the control of the bench more user-friendly.

\section{High-level control tools for calibration and analysis}

\subsection{MYST}

The MCAO Yorick Smart Tool (MYST) interface is the real "brain" of GeMS. With it, we can control each GeMS component, including the RTC within the Canopus bench, using EPICS commands. Figure 2 shows the Calibration $\mathcal{F}$ Control panel for the current release of the software, where both LGS and NGS loops as well as the TTM are controlled along with several other parameters for the wavefront reconstruction process. This panel allows the user to load optimal command matrices, as well as to choose disturbances, offsets to be added to the slopes calculations and different extrapolator models for the non-active actuators. Also in this panel the user has status displays and the option of running several calibration sequences, which are described below. The visualization of the current state of the loop as well as some statistical analysis can be done through the Real Time Display (RTD), also shown in the figure. The detailed description of how this GUI works is out of the scope of this paper, so we concentrate below only on the tools which are useful from the calibration point of view.

\subsubsection{Calibration sequences}

A complex instrument like Canopus has to be fine-tuned opto-mechanically, and the purpose of the calibrations sequences is to provide calibrations and settings for the optimal performance of the RTC. Canopus is mainly calibrated with the help of a simulation tool called yao, an adaptive optics package 

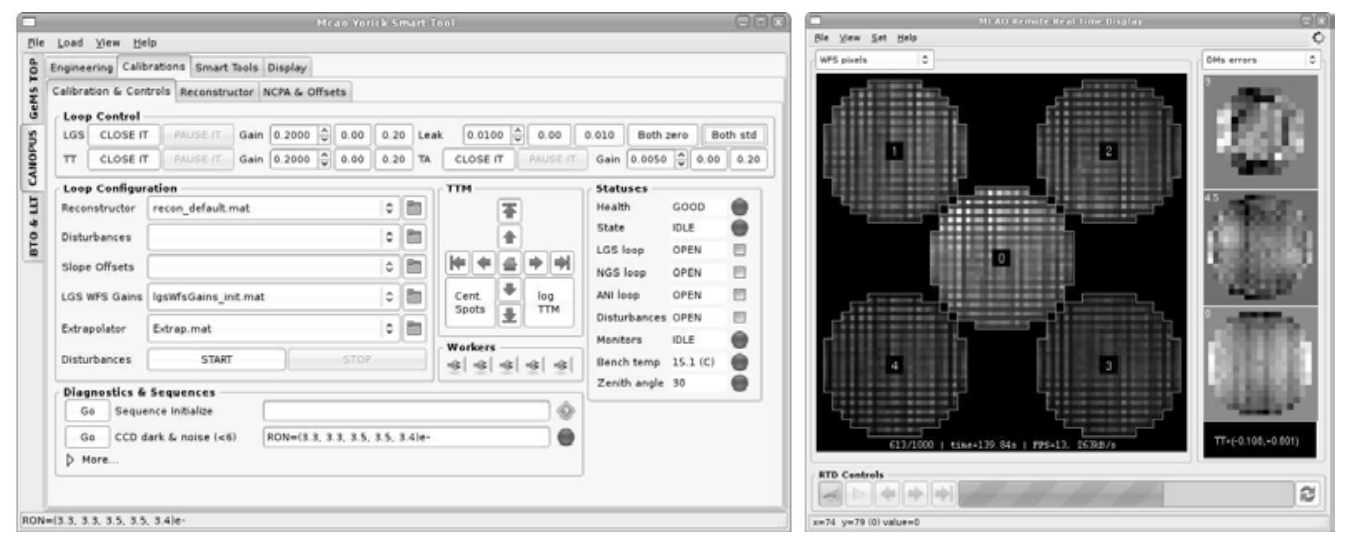

Fig. 2. Snapshot of MYST Calibration $\mathcal{E}$ Control panel for Canopus (left) and its visualization tool RTD (right).

written in yorick by F. Rigaut (http://www.maumae.net/yao), taking into account measurements of the system components, such as the mirror influence functions, CCD noise characteristics, etc.

Most of the calibration sequences for the LGS and NGS WFS have already been written in the framework of MYST, and in the following items we briefly describe how they work. They are to be further improved to take into account the integration with the other GeMS systems and the on-sky mode of operation.

- CCD dark \& noise: This sequence is the lowest level one. It acquires an adjustable number of non-illuminated frames and computes the average and rms over this set, leading to an estimate of the dark and readout noise per CCD, respectively. Each LGS WFS frame has simultaneous readouts for each of the $4 \mathrm{E} 2 \mathrm{~V} \mathrm{CCD}$ quadrants in which it is sub-divided, as set by the SDSU CCD controller. This reduces the readout time per pixel to $4 \mu s$ and delivers an average readout noise of about $3.5 \mathrm{e}$-, in perfect agreement with values specified by the RTC vendor. By now, the acquisition of frames is done after turning off the LGS and NGS calibration sources, and the lights in the clean room, but on-sky mode the Canopus entrance shutter will be closed for running this sequence.

- Initialization sequence: This sequence, as the name suggests, puts the system in an initial state, running the $\mathrm{CCD}$ dark \& noise sequence, loading the proper files and parameters (e.g. default reconstruction matrix, offsets, non-active actuators extrapolation model, loop gains and leakages), making sure that all loops are open, LGS calibration sources are on, etc. This sequence is run at the beginning of each of the following sequences described below.

- Registration sequence: An optimal registration of the DM0 actuators to the LGS WFS lenslets in crucial to get the most out of the dynamical range of the wavefront sensor. This sequence loads a reconstructor matrix that only acts on DM0 for a given LGS WFS path at each time, with the TTM being centered for this setup. After setting the loop gain and leakage instantaneously as zero, which means that no new commands are sent to the actual DM0 position by the reconstructor, it pokes 9 symmetrically distributed actuators on DM0 and computes the average slope deviations in $\mathrm{X}$ and $\mathrm{Y}$ directions, as well as any rotation and magnification detected in the WFS under analysis. The final output gives the average statistics of these deviations for each of the five beams, as well as the average statistics for all WFS paths. These deviations can then minimized by tuning the LGS WFS zoom and translation stepper motors that conjugate and align the DM0 pupil to the LGS WFS lenslets.

- Actuators gain sequence: This sequence has the purpose of calibrating the yao simulated influence functions for the 3 deformable mirrors taking into account measurements of the 5 LGS WFS paths. It loads control matrices to control individually each DM at a time without filtering the TTM contribution. In doing so, a tip and tilt of +100 mas around the flat position are induced with the TTM , and the DM under analysis tries to compensate these aberrations through its actuators. By 
recording the set of commands necessary to flatten the mirror one can estimate the factors with which to calibrate the micron-per-volt definitions applied by the parameter file built by yao.

- LGS centroid gain sequence: This sequence calibrates the quad-cell transfer functions of the LGS WFS. The user is asked if he/she wants to start the calibration from scratch, i.e., adopting a unity centroid gain and zero offsets, or just compare with the ones loaded already in the RTC. Under paused loop, this sequence uses the TTM to induce a series of increasing tips and tilts around the flat position, under paused loop. The measured slopes for each tip-tilt are recorded and fit in $\mathrm{x}$ and $\mathrm{y}$ by a $\tan ^{-1}$-like expression to obtain the transfer function, which can be visualized through the RTD by passing the mouse over different sub-apertures. Since the centroid gain under on-sky mode will vary with the atmospheric conditions, this parameter will be optimized by the Fast-Steering Array (FSA), a set of 5 mirrors set to rotate at a rate of $100-200 \mathrm{~Hz}$, and which will also be responsible for keeping the LGS asterism at the fixed configuration on the sky.

- NGS centroid gain sequence: This sequence currently uses three off-center NGS source stars for the calibration of the centroid gain of the NGS probes, and the calibration is made on an average basis. Once the best positions of the probes are determined in a semi-automatic way, the sequence is run more or less the same way as for the LGS centroid gain calibration, by fitting a theoretical function to the slopes measured versus the perturbation induced. This sequence is being completely automatized at this moment, extending the configuration of the NGS sources to other positions in the FoV. On sky, the centroid gains for the NGS WFS probes will have to rely on look-up tables for the atmospheric conditions estimated from the real time data.

A "MCAO Daily Calibration Log" is maintained in the intranet MCAO documentation webpage where the results of all the sequences are automatically posted, as well as the parameters loaded for that particular run. Also, in order to facilitate the analysis of dependencies with certain parameters such as temperature/flexure, the results are also logged inside our mcao-rtc directory in a format which is easily handled by plotting routines.

\subsection{Circular Buffer Analysis Tool}

The RTC allows the user (through MYST) to save circular buffers (CB) for posterior analysis of the system behavior and wavefront reconstruction. This includes saving slopes, covariance matrices, M1 \& M2 offloads, etc. A tool is being developed to allow the detailed analysis of the time series of some important variables, where the user will be able to identify possible problems occurred during a determined closed loop. This CB tool has been useful also in identifying some vibrational modes of the bench through a PSD analysis of the slopes, in the range of 0 to $400 \mathrm{~Hz}$. The re-design of the cooling system relies significantly on the vibrational frequencies induced by the cooling fans to be attached to the $-\mathrm{X}$ side of the bench, where the DMs HV power supplies are located.

\subsection{WAY and the diagnostic wavefront sensor}

The diagnostic wavefront sensor (DWFS), seen on figure 3, is a laboratory tool devised to help in the characterization and improvement of the performance in the science path. Two temporary fold mirrors located inside and above the bench re-direct the F/33.2 science beam to a focal plane located some centimeters in front of this instrument, and with the help of the collimating tube seen in the picture a lenslet array forms a Shack-Hartmann image of $24 \times 24$ sub-apertures on a CCD Pulnix camera. By moving this assembly in X, Y, tip and tilt we are able to locate the corresponding DWFS images of the NGS calibration sources in the field. The software Wavefront Analysis with Yorick (WAY) was developed in order to acquire and analyze WFS images of these calibration sources, delivering the decomposition in Zernike modes, Strehl and rms information. The Zernike coefficients are saved in the software directory and used by the MYST tools to build non-common path aberration (NCPA) files which contain slope offsets to be used by the LGS WFS. When combining the aberrations obtained in several points, NCPA offsets can be computed for the MCAO system geometry through tomographic techniques (see [2]). Using this technique after 1 or 2 iterations Strehl ratios above 95\% (H-band) in the 
Table 1. Recent results from the tomography applied to build NCPA files for the science path (H-band), with and without taking the astigmatism contribution in the error budget (the main source of static aberrations in this path). Positions numbered 0-4 correspond to the 5 NGS positions coincident with the LGS asterism ( 0 at the center); the remaining positions are inner NGS calibration sources, separated from the central source by 21.4 arcsec. Average and rms results are $96 \%$ and $1 \%$ (for both with and without astigmatism cases), totally inside the design specifications.

\begin{tabular}{lccccccccc}
\hline Position & 0 & 1 & 2 & 3 & 4 & 5 & 6 & 7 & 8 \\
\hline Strehl (w/ astig) & $95.0 \%$ & $95.8 \%$ & $94.4 \%$ & $95.9 \%$ & $96.6 \%$ & $97.0 \%$ & $95.5 \%$ & $96.9 \%$ & $95.8 \%$ \\
Strehl (w/o astig) & $97.3 \%$ & $96.7 \%$ & $95.9 \%$ & $95.9 \%$ & $96.6 \%$ & $97.0 \%$ & $95.5 \%$ & $96.9 \%$ & $95.8 \%$ \\
\hline
\end{tabular}
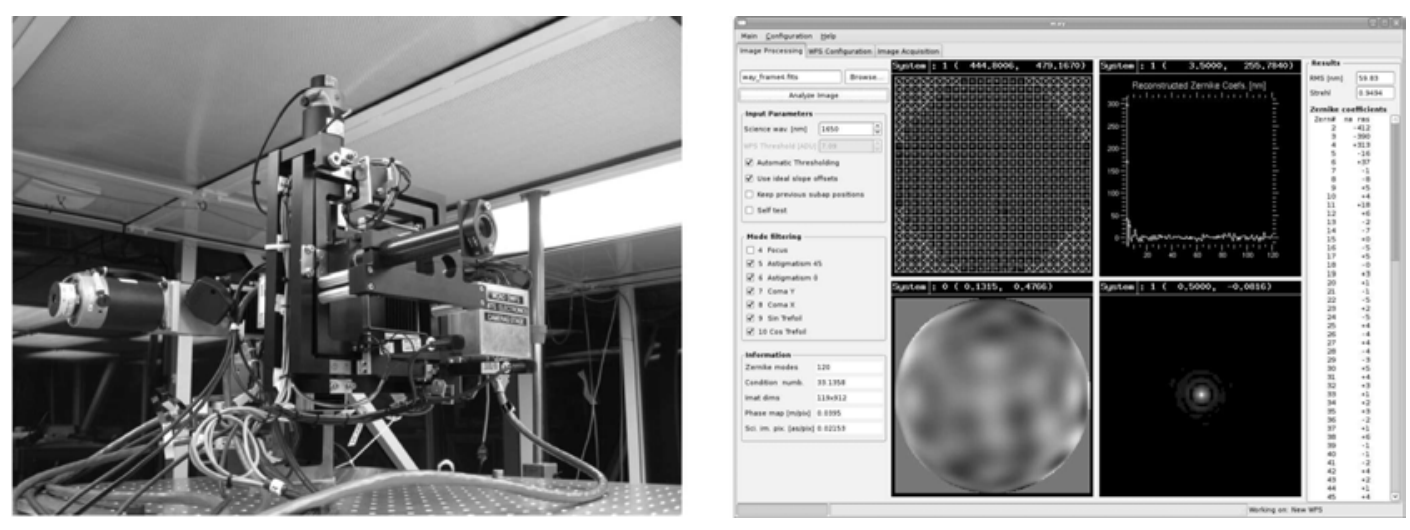

Fig. 3. Diagnostic wavefront sensor temporarily mounted on the top of the bench (left) and a snapshot of the WAY analysis panel (right).

whole field and uniformity equal or better than $2.6 \%$ (peak-to-valley) inside the distribution of the LGS asterism are obtained, as shown by a recent work (see table 1). Notice that better results are probably prevented to be achieved due to aberrations likely to be present in the non-common path components, like the fold mirrors and the DWFS optics itself. This could possibly happen because none of these elements are conjugated to the altitudes of the DMs. Final NCPA files will futurely be obtained with the GSAOI and a plate of $4 \times 4$ sources for calibration, but the current laboratory results are already optimistic regarding to what to expect in operational mode. The DWFS is now being upgraded to match its final design, in which a direct imaging mode will be used to evaluate point spread function (PSF) variations across the field; this is accomplished by the use of an SBIG ST-402 CCD camera and a barlow lens for magnification of the image formed in the focal plane.

\section{Bench optimization and problems still to be mitigated}

There is still a whole series of tasks to be conducted in order to improve the bench performance. This includes alignment procedures, optimization of the neutral position for the science ADC, among others. At this moment, the servo motors that conjugate the simulated zenith angle of the LGS calibration source to the LGS WFS assembly are almost fully integrated. The reliability on the positioning of the LGS WFS stepper motors was checked by taking measurements of DM0-lenslet pupil registration when moving the bench between 0 and 54 degrees along 50 cycles. The motors performed reliably and under specs in those tests, keeping their average mis-registrations (see section 2.1.1) below 4\% (peak-to-valley) of a sub-aperture in all beams. However, flexure tests between 0 and -54 degrees are still pending.

Focusing of the NGS WFS assembly has recently been performed, leading to a NGS spot size of about 0.3-0.4 arcsec in all three probes when using a diffraction-limited source in the calibration plate. 

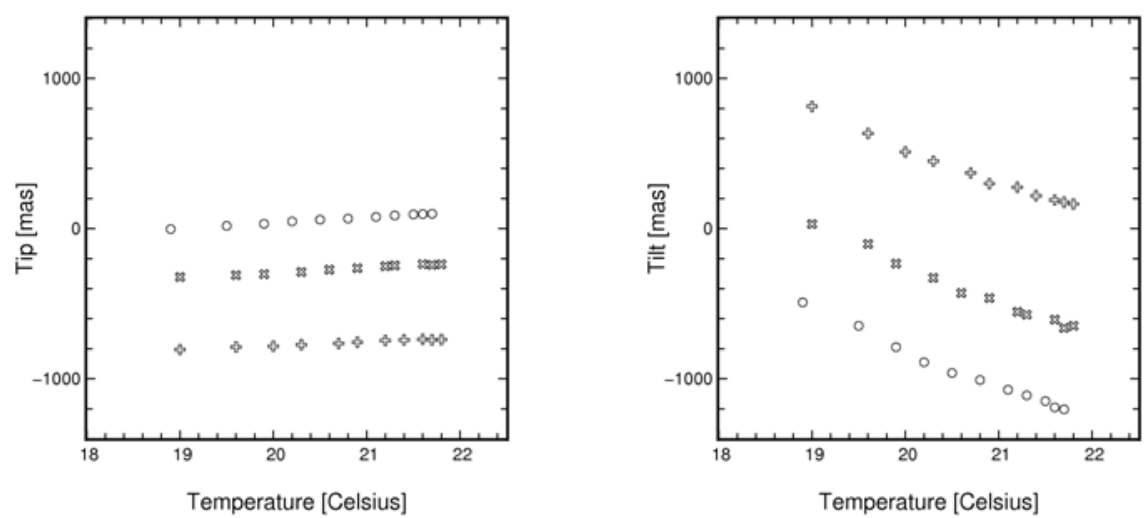

Fig. 4. Thermal drift of the TTM. These data were acquired during the acceptance tests for the LGS WFS motors, and symbols refer to bench rotated to $0^{\circ}$ (circle), $30^{\circ}$ ("x") and $54^{\circ}$ (cross) in the -X direction.

However, a fine-tuning of the ADC in the NGS WFS path has still to be done. This will allow us to evaluate if the hints of elongation we noticed during the tests are due to a bad setup of the ADC zeropoint or to some mis-alignment of the quad-cell reflective pyramid inside the probes, which couples the beam image to $300 \mu \mathrm{m}$ fibers. The relative throughput of these fibers and APDs has also been measured, showing that there are some non-uniformity problems in the APDs. The absolute throughput from the calibration source to the NGS probes was recently measured, and will be re-done when DM0 is back from fixing a defective actuator and its coating. This will provide the answer to the question on the magnitude of natural guide stars necessary for the MCAO system to work under the specifications.

At last, some tests are scheduled in order to verify which component(s) of the bench is(are) responsible for the thermal drift observed in the TTM (see figure 4). This problem so far precludes the utilization of the TTM for centering the LGS WFS when operating above a certain range of temperatures $(\Delta T \approx 5$ degrees Celsius), given that the TTM dynamical range goes only from -1.4 to +1.4 arcsec in both directions. From figure 4, taken during the latest flexure tests, we see clearly this behaviour, especially in the tilt direction. We now plan to use a laser attached to the bench and aligned in a far-field component to track, with the help of a CCD camera, the displacements seen in different parts of the bench for a wide range of temperatures. This procedure will allow us to identify the component responsible for the thermal drift, and mitigate this problem.

AGR acknowledges the support of the European Communities Commission Seventh Framework Programme E-ELT Prep (Grant INFRA-2007-2.2.1.28), in the form of a travel grant which enabled her to attend this conference. The Gemini Observatory is operated by the Association of Universities for Research in Astronomy, Inc., under a cooperative agreement with the NSF on behalf of the Gemini partnership: the National Science Foundation (United States), the Science and Technology Facilities Council (United Kingdom), the National Research Council (Canada), CONICYT (Chile), the Australian Research Council (Australia), Ministério da Ciência e Tecnologia (Brazil), and Ministerio da Ciencia, Tecnologia y Innovación Productiva (Argentina).

\section{References}

1. Neichel B., Rigaut F., Gratadour D., Garcia-Rissmann A., this conference.

2. Rigaut F., Neichel B., Bec M., Boccas M., Garcia-Rissmann A., Gratadour D., this conference. 